\title{
BioNNA: the Biodiversity National Network of Albania
}

\author{
Michela Pacifici', Fabio Attorre², Stefano Martellos ${ }^{3}$, Ferdinand Bego ${ }^{4}$, \\ Michele De Sanctis ${ }^{2}$, Petrit Hoda ${ }^{5}$, Marjol Meço ${ }^{4}$, Carlo Rondinini', \\ Enerit Saçdanaku ${ }^{5}$, Elson Salihaj ${ }^{6}$, Edoardo Scepi ${ }^{7}$, \\ Lulëzim Shuka ${ }^{4}$, Andrea Ghiurghi ${ }^{8}$
}

I Global Mammal Assessment programme, Department of Biology and Biotechnologies, Sapienza Università di Roma, Rome, Italy 2 Department of Environmental Biology, Sapienza Università di Roma, Italy 3 Department of Life Sciences, University of Trieste 4 Tirana University, Faculty of Natural Sciences, Department of Biology, Tirana, Albania 5 Tirana University, Faculty of Natural Sciences, QKFF, Tirana, Albania 6 NaturAL, IPA 2013 - Natura 2000 and Protected Areas Torre Drin, Tirana, Albania 7 A.R.E.A. Gis s.r.l. - Ambiente Ricerca E Applicazioni, Rome, Italy 8 International Union for Conservation of Nature ECARO, Belgrade, Serbia

Corresponding author: Michela Pacifici (michela.pacifici@uniroma1.it)

Academic editor: S. Lengyel | Received 20 November 2017 | Accepted 20 February 2018 | Published 13 March 2018

http://zoobank.org/C48082C8-D7DC-436E-B906-3C3F65BBF1CC

Citation: Pacifici M, Attorre F, Martellos S, Bego F, De Sanctis M, Hoda P, Meço M, Rondinini C, Saçdanaku E, Salihaj E, Scepi E, Shuka L, Ghiurghi A (2018) BioNNA: the Biodiversity National Network of Albania. Nature Conservation 25: 77-88. https://doi.org/10.3897/natureconservation.25.22387

\begin{abstract}
Recently, the Albanian Government started the process to join the European Union. This process also involves matching the EU parameters in protecting its biodiversity. In order to support the Albanian authorities, the Italian Ministry of Foreign Affairs, General Directorate for Development Cooperation (DGCS) and the International Union for Conservation of Nature (IUCN) joined efforts in the project "Institutional Support to the Albanian Ministry of Environment, Forest and Water Administration for Sustainable Biodiversity Conservation and Use in Protected Areas". This project aims at identifying priority needs in safeguarding ecosystem services and biodiversity conservation. Another project funded by the EU - "Strengthening capacity in National Nature Protection - preparation for Natura 2000 network" started in 2015 with the aim to raise awareness for assisting local and national Albanian institutions to better exploit the potential of protected areas. One of the main issues encountered during these projects was the need for a national biodiversity data repository. The Biodiversity National Network of Albania (BioNNA) has been created to aggregate occurrence records of plants and animals and aims at becoming the most relevant source of information for biodiversity data as far as Albania is concerned. In this paper, the authors detail structure and data of BioNNA, including the process of data gathering and aggrega-
\end{abstract}

Copyright Michela Pacifici et al. This is an open access article distributed under the terms of the Creative Commons Attribution License (CC BY 4.0), which permits unrestricted use, distribution, and reproduction in any medium, provided the original author and source are credited. 
tion, taxonomic coverage, software details and WebGIS development. BioNNA is a milestone on the path towards Albania's inclusion in the EU and has also a relevant potential social relevance for improving people's awareness on the importance of biodiversity in the country.

\section{Keywords}

Albania, biodiversity, database, occurrence records, species

\section{Introduction}

Recently, the Albanian Government started the process to match European Union parameters in biodiversity conservation, as part of a larger process which is expected to lead to its inclusion in the Union. This process is highlighted by the recent expansion of the Albanian protected area system, as well as by the efforts to gather and aggregate biodiversity data (fundamental for reporting to the European Union and to other international conventions). In order to support the Albanian authorities, the International Union for Conservation of Nature (IUCN) implemented a project funded by the Italian Ministry of Foreign Affairs, General Directorate for Development Cooperation (DGCS), entitled "Institutional Support to the Albanian Ministry of Environment, Forest and Water Administration (currently Ministry of Environment - MoE) for Sustainable Biodiversity Conservation and Use in Protected Areas" (hereafter the "Institutional Support" project). This five-year project aimed at identifying priority needs in safeguarding ecosystem services and biodiversity conservation. The project focused on two protected areas (PAs), the Buna River Protected Landscape and the Shebenik-Jabllanicë National Park, which were selected, amongst other criteria, for the relevance of the ecosystem services they provide to local communities. The objective of the project was to start a capacity building process, which would lead the MoE to adopt a systemic approach in planning and managing protected areas. This included inter alia the participatory preparation of the two PAs management plans, training of local MoE staff, NGOs and university post graduate students on ecological monitoring, implementation of concrete activities (such as building and renovation of PAs ecotourism facilities) and carrying out awareness-raising actions.

Another project funded by the EU, "Strengthening capacity in National Nature Protection - preparation for Natura 2000 network" (NaturAL), is being implemented in Albania since 2015, aiming at a) raising awareness for assisting local and national Albanian institutions to better exploit the potential of protected areas and b) further promoting the partnership between the Italian Cooperation and the Albanian Ministry of Environment. Its main objective is to stop the loss of biodiversity in Albania by further improving the management practices of protected areas. This will be achieved by implementing five PAs' Management Plans and by preparing a preliminary list of Natura 2000 sites, with a view to their future submission to the European Commission by the Albanian authorities. A proper organisation and maintenance of, confidence on and accessibility to biodiversity data is mandatory for elaborating conservation policies 
and strategies, performing strategic assessments and identifying priorities of interventions, hence reducing the current rate of biodiversity loss (Wheeler et al. 2012, Costello et al. 2013). Especially since the Earth Summit of Rio in 1992, electronic access to biodiversity information has become a priority task worldwide, leading to great progress in the field of Biodiversity Informatics (European Commission 2011, Martellos and Attorre 2012, Costello et al. 2013). This, amongst other relevant results, led to the creation of global federated networks for the aggregation of biodiversity data (e.g. the Global Biodiversity Information Facility (GBIF) - http://www.gbif.org/ - and the BioCASE - http://www.biocase.org (Holetschek et al. 2012)). The GBIF especially, which aggregates more than 750 million occurrence data, was developed to accomplish one of the aims of the Convention on Biological Diversity, i.e. to make biodiversity data accessible online.

Hence, in the framework of the "Institutional Support" project, one of the strategic needs expressed by the Albanian institutions was the development of a national biodiversity data repository to aggregate, manage and make data available online. Such a tool was seen as mandatory for supporting Albanian Institutions in elaborating effective conservation strategies and reporting to relevant EU Conventions. Too often biodiversity data are unavailable to institutions, researchers and the general public in many countries or, if available, they are scattered in several different repositories which differ in platform, structure and data semantics (Edwards et al. 2000, Schuurman and Leszczynski 2008, Heidorn 2011, Martellos and Attorre 2012). Furthermore, biodiversity data are often collected in the framework and with the technical and financial support of international projects. Unfortunately, in absence of official data repositories, these data tend to disappear at the project's end. Several international projects have also a limited impact, since they are unrealistically complicated and impossible to maintain with limited local funding and human resources (Šašić et al. 2015). Furthermore, biodiversity data collected in extemporaneous efforts are often incomplete, with gaps for many taxa and habitats (Qiriazi and Sala 2000, Mullaj et al. 2007).

Albania has maintained many of its traditional agrosilvicultural practices, thus preserving most of its rich biodiversity, also in the absence of a formal system of protected areas. However, a process of over-exploitation of natural resources started in the last decades (since the country opened to external influences (Šašić et al. 2015)), together with the need for achieving several targets mandatory for being part of the EU, prompted the creation of an official network of protected areas. However, planning the development of an effective network of protected areas requires a thorough knowledge of local biodiversity. While Albania hosts a rich biodiversity (Qiriazi and Sala 2000, Mullaj et al. 2007, Mizsei et al. 2017, Szabolcs et al. 2017), only few data exist in the digital domain (e.g. Corine Biotopes (https://www.eea.europa.eu/data-and-maps/data/clc2012-raster), Emerald (http://cdr.eionet.europa.eu/al/coltswjhw/Emerald/envtncdca), Red Lists of Flora and Fauna of Albania (http://www.nationalredlist.org/files/2015/06/ Red-list-of-Albanian-flora-and-fauna-2013-MO-1280-20-11-2013.pdf)) and are often difficult to access, mainly due to scarcity of ICT resources and the absence of long-term vision and strategy in the past. Hence, it was planned to transfer to Albania previous 
experiences (Martellos et al. 2011, Holetschek et al. 2012), aiming at the development of a sustainable infrastructure for the aggregation of biodiversity data, the Biodiversity National Network of Albania (BioNNA). BioNNA was developed for supporting the National Biodiversity Strategy and Action Plan of the Albanian MoE for the period 2000-2015 and it will serve as a data repository according to international standards of biodiversity data collected by future projects as well as from citizen science initiatives. The Action Plan defines the main directions for preserving biodiversity and habitats, the identification and institution of protected areas and the protection of species inside and outside these areas. Due to BioNNA, biodiversity data collected from different sources are made available in a standard format to institutional decision-makers and planners, researchers, eco-tourists and the general public. Data originating from several different initiatives and projects are aggregated and validated by using standardised procedures by national and international experts in botany, zoology and biodiversity informatics.

In the present paper, the architecture of BioNNA, the data it currently aggregates and its WebGIS platform are detailed.

\section{Methods}

\section{Data and taxonomic coverage}

BioNNA aggregates primary biodiversity data, i.e. occurrences of plants and animals recorded in Albania. They have been obtained by Albanian and international researchers from three different sources: i) field data collected in the years 2015-2016 during the Institutional Support and the NaturAL projects, ii) data published in the scientific literature and iii) field data collected in the last 10 years by international experts or local researchers involved in the Institutional Support and the NaturAL projects. Field data have been collected mostly during June-October 2012/2013/2016 field campaigns in 7 protected areas, which were selected because of their relevance in terms of species and habitats diversity: Buna River-Velipoje Protected Landscape, Shebenik-Jabllanica National Park, Lake Shkodra Managed Nature Reserve, Korab-Koritnik Managed Nature Reserve, Divjaka-Karavasta National Park, Tomorri Mountain National Park, and Bredhi i Hotoves-Dangelli National Park (Fig. 1).

While a detailed description of the methodologies applied during field work is beyond the scope of this paper, it is important to mention at least the approaches used for each taxonomic group. Botanical data have been collected according to the phytosociological methods of Braun-Blanquet (1964) and Westhoff \& van der Maarel (1978). The list of species occurring in scattered plots, selected in relation to the homogeneity of physical features, vegetation structure and species dominance, has been reported in order to identify vegetation communities and habitats.

During faunistic surveys, fish population sampling was undertaken following the CEN 14757 standardised protocol, using benthic multi-mesh gillnets and pelagic multi-mesh gillnets. For amphibians and reptiles, Visual Encounter Surveys (VES) 


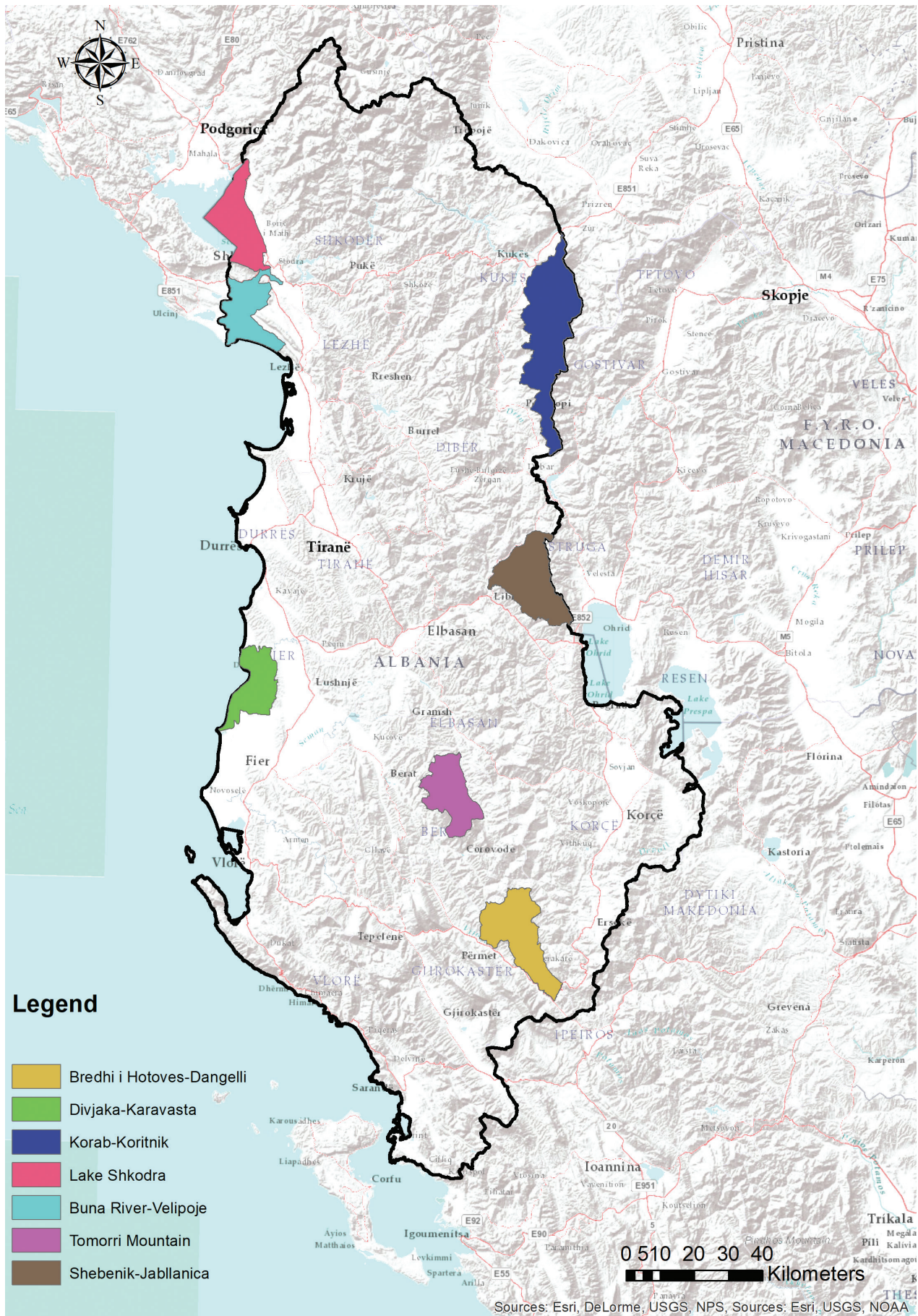

Figure I. Distribution of the 7 protected areas in which field campaigns have been undertaken during the "Institutional Support to the Albanian Ministry of Environment, Forest and Water Administration for Sustainable Biodiversity Conservation and Use in Protected Areas" and NaturAL projects. 
have been applied through transects according to the habitat requirements of each taxon. The censuses of birds were undertaken by using binoculars, bird calls and spotting scopes. Counting points were chosen as a function of accessibility, visibility and the best coverage of the area. Four different methods have been used for mammals: 1) Sherman traps, for capturing live specimens of small terricolous mammals, 2) camera traps, located in selected sites to sample large and medium-sized mammals, 3) tracks and signs observed along transects and 4) mist nets and bat detectors to study the presence of bats, mostly in caves and disused bunkers.

In Albania, 411 species of plants and 1206 species of animals are listed in the IUCN Red List of Threatened Species (Zeneli et al. 2014). Although the country is known to host 3629 species of plants and 1751 of animals (Meço and Mullaj 2015, Olšavská et al. 2016), these figures are likely to increase, since some areas in the Albanian territory are largely unexplored (Olšavská et al. 2016), especially in the north- and south-eastern portions of the country and some taxa, particularly animals, are still understudied even in well-known areas (Bego et al. 2018). To date, BioNNA has aggregated 9967 occurrence records of plants (1452 infrageneric taxa) and 11376 occurrence records of animals (637 infrageneric taxa). Although insects are known to be the largest group of animals on the planet, the number of records for this taxonomic group (266, 93 infrageneric taxa) are very scarce, mostly because of a lack of local entomologists.

\section{Software}

The core of BioNNA is a federated database system of primary biodiversity data (i.e. data obtained from floristic and faunistic observations and from specimens of natural history collections), which uses the BioCASE protocol for allowing the communication amongst the nodes of the federation and $\mathrm{ABCD}$ as data standard (Martellos et al. 2011, Holetschek et al. 2012, Martellos and Attorre 2012). When a user queries the system, the output is produced by sending the query to all federated nodes and returning an answer in the standard format $\mathrm{ABCD}$. The BioCASE provider software is a free package supplied by the BioCASE project (http.//www.biocase.org), supported by the Botanic Garden and Botanical Museum of Berlin-Dahlem. The Web-App was the part of the system which required the highest effort, since it was developed from scratch. It allows the use of BioNNA in two different modalities; as a Standard User, which is allowed to query and retrieve data and as a Professional User, which also has access to several editing tools for managing the databases. In order to improve users' experience, the Web-App was developed by using innovative libraries from DEVEXPRESS, within a Microsoft Visual Studio development environment. All the functions of the Web-App are provided with automated input control tools, in order to prevent any erroneous use, in particular in data cleansing and updating processes.

BioNNA is hosted in a web portal which, other than the Web-App, hosts:

- Introductive Pages, where information about BioNNA and the dataset can be retrieved; 
- Species Fact Sheets, where it is possible to list the taxa and their biological features. This section also links to the Encyclopaedia of Life (EOL), for retrieving information on taxonomy, distribution and conservation status of the species;

- Species Data pages, where it is possible to list the occurrence points. From the Species Data page, it is also possible to access the Web-GIS viewer;

- Web-GIS viewer, which is used for visualising the spatial distribution of each infrageneric taxon. This tool has been specifically developed for the BioNNA system, making use of open source and open code tools;

- Protected Areas page, which contains Socio-Ecological Fact Sheets (data and technical information) about the current situation of the Albanian protected areas.

A well as being a federated network of data providers, BioNNA was also planned as a data provider for the BioCASE and GBIF networks, in order to make the biodiversity heritage of Albania available worldwide.

\section{Results and discussion}

\section{BioNNA: datasets and policy}

BioNNA is currently the largest aggregator of primary biodiversity data in Albania and it is planned to grow further by aggregating new datasets. However, the aggregation of a new dataset into the federation is not an automated process. Each dataset must have a certain level of data quality, as well as a minimum mandatory set of data: a unique ID for each observation record, the scientific name of the taxon, latitude and longitude. For consistency purposes, it is suggested that the coordinates follow the WGS84 geographic system in decimal degrees. These minimum requirements are evaluated by the Scientific Committee of BioNNA. In addition to the minimum mandatory dataset, many other data can potentially be included. Those which have been already implemented in the Web-App of BioNNA are: date of survey or collection, collector or observer, locality of collection or observation, community in which the organism was surveyed and references. Furthermore, the use of ABCD, which lists ca. 1200 concepts, permits far more data for each observation record to be aggregated in the BioNNA federation.

BioNNA is also storing data on sensitive taxa. In this case, a relevant issue is given by showing the exact locations of occurrence, providing the risk of misuse of those sensitive data by parties with commercial interests. On the other hand, the exact location of sensitive taxa (e.g. species classified as threatened by the IUCN - critically endangered, endangered or vulnerable - e.g. important nursery colony of bats, den site of bears, otter holts etc.) is an important source of information for decision-makers, such as the Ministry of Environment, the National Environmental Agency (NEA), the National Agency of Protected Areas (NAPA) and other Ministries and development agencies. Hence, sensitive data will not be displayed online, but provided under strict safeguard policy, in order to avoid their misuse. 
Currently, BioNNA aggregates not only data of taxa listed in the Birds and Habitats Directives, but also of several other taxa and potentially of all those which are known to occur in the country. This is of pivotal importance for Albania, since the data aggregated in BioNNA could provide evidence of the need for protection of some endemic/rare taxa or highlight areas of high biodiversity or relevance, deserving some degree of protection.

\section{Using BioNNA}

The web portal of BioNNA (www.bionna.al) provides users with several ways to access the data. The query system hosts two tools, one for plants and one for animals. In the 'Species data' section, in addition to the data sheets, users can visualise a data table with all the occurrence records for a species. For each occurrence, ID number, latitude and longitude, as well as the optional details are reported (when available: date of data entry, number of individuals, type of data collected, references, vegetation community (only for plants) and locality in which the individual has been found; Fig. 2). In this section, the user can also create a spatial reference file (.shp) for each selected species and visualise it by using the WebGIS viewer, which is one of the most relevant features of BioNNA. It displays occurrence points on a satellite map of Albania, evidencing protected areas, as well as counties borders (Fig. 3). From the 'Species data' page, it is possible, using the button "Add Species to Map", to load the occurrences of a taxon into the Web-GIS Viewer. This can be done for a single taxon or for a selection of taxa. The viewer displays the points on the map, hence allowing a preliminary analysis of the spatial distribution of the taxa. Furthermore, it is possible to access the metadata associated with the shapefile of each taxon. From the 'Species data section' it is also possible to download a data table of occurrence records in Excel format (.xls), which was chosen since it can be imported into several modelling and analysis tools (e.g. MaxEnt (Elith et al. 2011) and GeoCAT (Bachman et al. 2011)).

\section{Relevance of BioNNA and future development}

BioNNA aims at supporting Albanian Institutions in managing data on the biodiversity of the country. Several stakeholders will potentially benefit from it, especially whether it will receive regular updates and grow with the aggregation of other datasets. Practitioners will benefit from knowing which taxa are known to occur in the areas they manage, in order to plan species- or habitat-specific conservation measures. A detailed and up-to-date knowledge of the distribution of "problematic" taxa, e.g. those which prey on livestock (e.g. wolf, bear, fox, jackal) will help reducing the conflicts between human needs and wildlife conservation. Depicting the areas in which charismatic species live will also improve tourism, providing economic benefits to local communities, hence leading to an increased awareness of the values provided by wildlife conservation amongst local populations. 


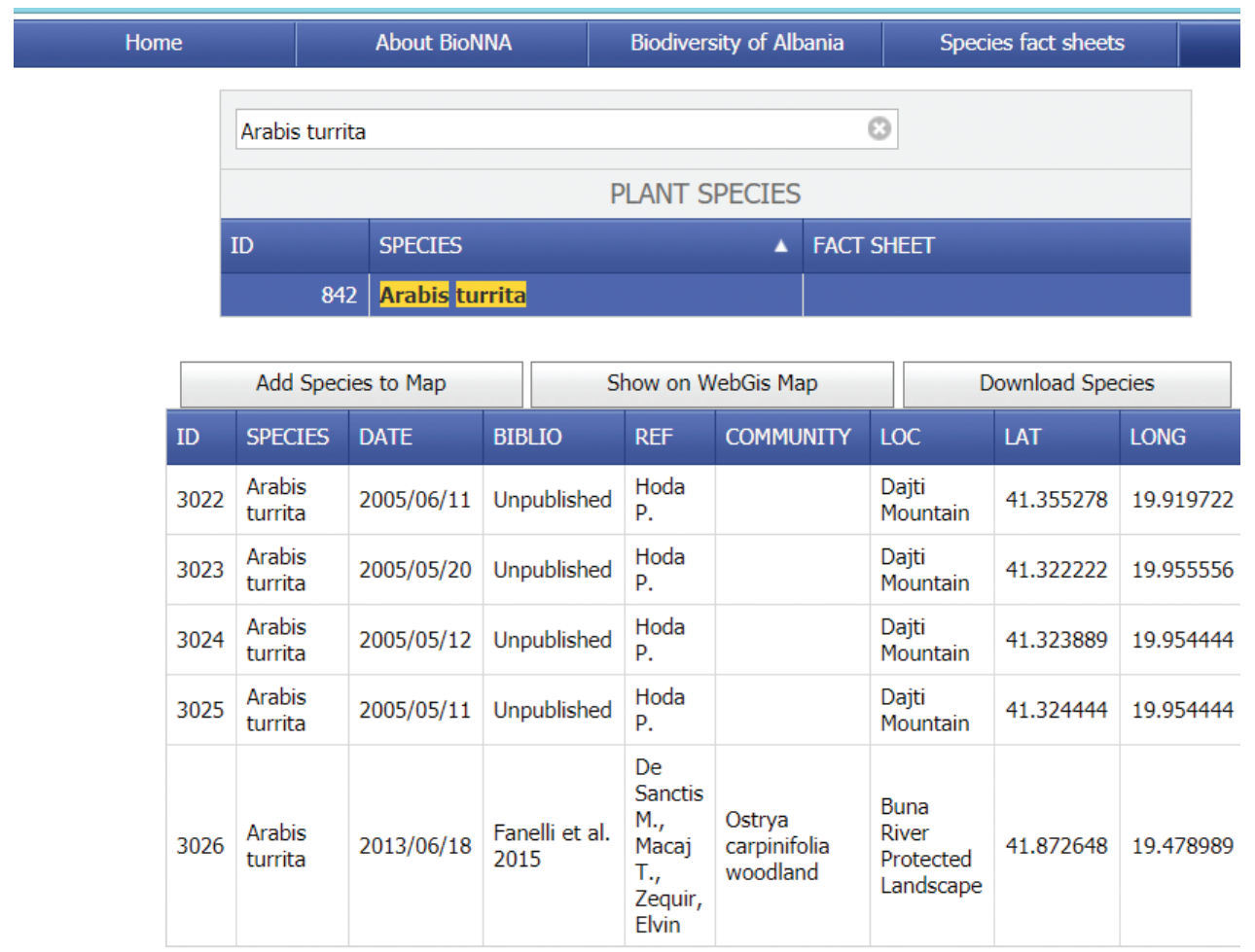

Figure 2. The BioNNA search interface.

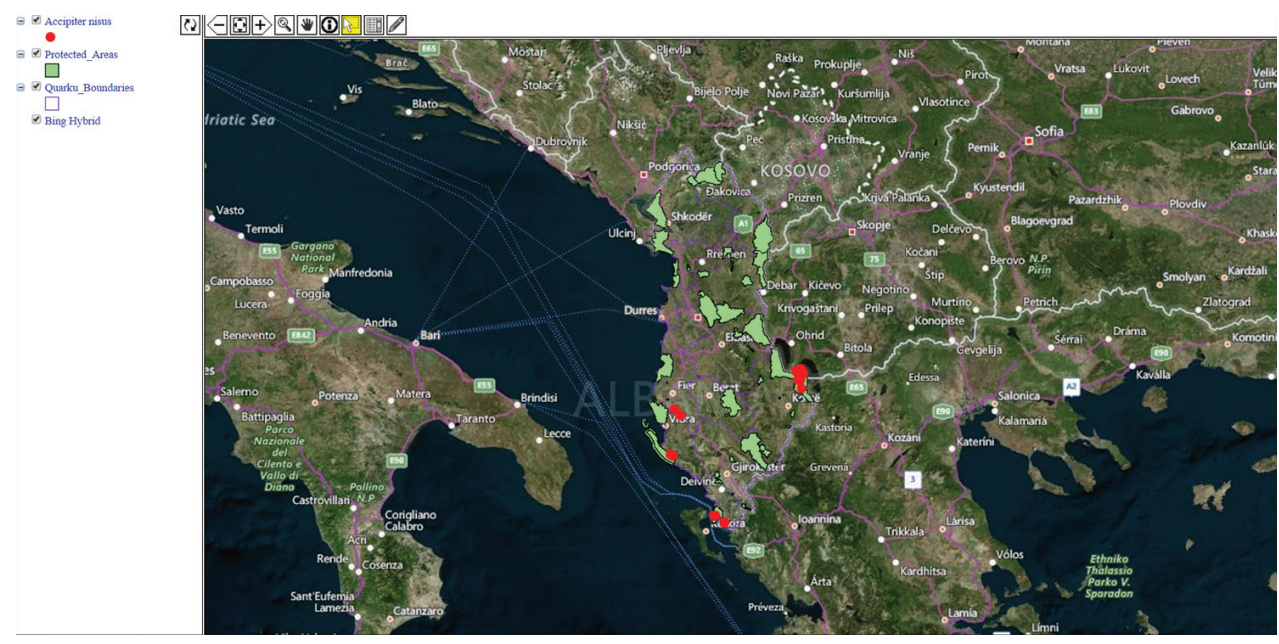

Figure 3. BioNNAWebGIS interface. 
BioNNA will also be useful to scientists in depicting gaps in knowledge of the biodiversity of Albania, hence driving new sampling efforts. A large portion of Albania still remains under-investigated and it is likely that several interesting/rare taxa occurring in the country are still to be reported (Bego et al. 2018). Academics will have a fundamental role in the development of BioNNA, since they will be involved in supporting the Albanian Ministry of Environment for its future growth. Therefore, a pool of outstanding local researchers has been selected as the Scientific Committee of BioNNA, with the aim of:

1. Validating the dataset before aggregating them in the federated database system;

2. Deciding whether to provide data on sensitive species to interested parties. Monitoring the use of the sensitive data should be mandatory, in order to ensure the protection of sensitive taxa;

3. Promoting the use of BioNNA to elaborate effective conservation strategies and defining the main strategies for its further development (i.e. through the implementation of citizen science initiatives - e.g. initiatives such as the CSMON-LIFE project, BioBlitzes, The Great Nature, Project BudBurst, Nature's Notebook (Van Vliet and Moore 2016).

The Scientific Committee will be supported by international scientists during its early stages. The active cooperation of local scientists with the Albanian Ministry of Environment will be fundamental to improve BioNNA and make it a reliable reference point for Albanian biodiversity data. Other projects (e.g. "Conservation and sustainable use of biodiversity at Lakes Prespa, Ohrid and Shkodra/Skadar") currently aim at monitoring wild species in Albania and the occurrence data collected by scientists and the staff of protected areas may well be integrated within the BioNNA database.

\section{Acknowledgments}

This study was carried out within the framework of two projects: the IUCN Project "Institutional Support to the Albanian Ministry of Environment, Forest and Water Administration for Sustainable Biodiversity Conservation and Use in Protected Areas" funded by the Italian Ministry of Foreign Affairs, Directorate General for Development Cooperation and the "Strengthening capacity in National Nature Protection - preparation for Natura 2000 network" (NaturAL) project, funded by the European Commission. We also thank all the experts who contributed to the BioNNA development, in particular Caterina Carugati, Genti Kromidha, Taulant Bino, Ermelinda Gjeta, Bledi Hoxha, Ermelinda Mahmutaj, Ajola Mesiti, Alfred Mullaj, Arben Palushi, Francesca Pella, Spase Shumka, Philippe Théou, Vito Emanuele Cambria, Simone Stirpe and Denise Kosanke and the staff of the visited protected areas. 


\section{References}

Bachman S, Moat J, Hill AW, de la Torre J, Scott B (2011) Supporting Red List threat assessments with GeoCAT: Geospatial conservation assessment tools. Zookeys 150: 117-126. https://doi.org/10.3897/zookeys.150.2149

Bego F, Saçdanaku E, Pacifici M, Rondinini C (2018) Small terrestrial mammals of Albania: distribution and diversity (Mammalia, Eulipotyphla, Rodentia). ZooKeys 742: 127-163. https://doi.org/10.3897/zookeys.742.22364

Braun-Blanquet J (1964) Pflanzensoziologie. Grundzüge der Vegetationskunde (3 ${ }^{\text {rd }}$ edn). Springer, Vienna. https://doi.org/10.1007/978-3-7091-8110-2

Costello MJ, Michener WK, Gahegan M, Zhang Z-Q, Bourne PE (2013) Biodiversity data should be published, cited, and peer reviewed. Trends in Ecology \& Evolution 28: 454-461. https://doi.org/10.1016/j.tree.2013.05.002

Edwards JL, Lane MA, Nielsen ES (2000) Interoperability of Biodiversity Databases: Biodiversity Information on Every Desktop. Science 289: 2312-2314. https://doi.org/10.1126/ science.289.5488.2312

Elith J, Phillips SJ, Hastie T, Dudík M, Chee YE, Yates CJ (2011) A statistical explanation of MaxEnt for ecologists. Diversity \& Distributions 17: 43-57. https://doi.org/10.1111/ j.1472-4642.2010.00725.x

European Commission (2011) Final communication from the commission to the European parliament, the council, the economic and social committee and the committee of the regions: our life insurance, our natural capital: an EU biodiversity strategy to 2020. May 3, 201. Brussels.

Heidorn PB (2011) Biodiversity informatics. Bulletin of the Association for Information Science and Technology 37: 38-44. https://doi.org/10.1002/bult.2011.1720370612

Holetschek J, Dröge G, Güntsch A (2012) The ABCD of primary biodiversity data access. Plant Biosystems - An International Journal Dealing with all Aspects of Plant Biology 146: 771-779. https://doi.org/10.1080/11263504.2012.740085

Martellos S, Attorre F, De Felici S, Cesaroni D, Sbordoni V, Blasi C, Nimis PL (2011) Plant sciences and the Italian National Biodiversity Network. Plant Biosystems - An International Journal Dealing with all Aspects of Plant Biology 145: 758-761. https://doi.org/1 $0.1080 / 11263504.2011 .620342$

Martellos S, Attorre F (2012) New trends in biodiversity informatics. Plant Biosystems - An International Journal Dealing with all Aspects of Plant Biology 146: 749-751. https://doi.org/10.1080/11263504.2012.740092

Meço M, Mullaj A (2015) Phenological aspects of Albanian flora. Proceedings of International Conference On Soil. 04-06 May 2015, Agricultural University of Tirana, Tirana, Albania: Proceedings, 164.

Mizsei E, Jablonski D, Végvári Z, Lengyel S, Szabolcs M (2017) Distribution and diversity of reptiles in Albania: a novel database from a Mediterranean hotspot. Amphibia-Reptilia 38: 157-173. https://doi.org/10.1163/15685381-00003097

Mullaj A, Vangjeli J, Peçi D, Imeri A (2007) General considerations on the flora and vegetation of Albania's rivers. In: Pinna M, Felice Uricchio V, Michele Aresta M, Basset A (Eds) 
Rivers and citizens Cross-border experiences in environmental protection and sustainable development). University of Salento, 17-25.

Olšavská K, Slovák M, Marhold K, Štubňová E (2016) On the origins of Balkan endemics: the complex evolutionary history of the Cyanus napulifer group (Asteraceae). Annals of Botany 118: 1071-188. https://doi.org/10.1093/aob/mcw142

Qiriazi P, Sala S (2000) Environmental problems of Albania. In: Netherlands S (Ed.) Remote Sensing for Environmental Data in Albania: A Strategy for Integrated Management, 13-30. https://doi.org/10.1007/978-94-011-4357-8_4

Šašić M, Popović M, Cuvelier S, Đurić M (2015) Contribution to the knowledge of the butterfly fauna of Albania. Nota Lepidopterologica 38: 29-45. https://doi.org/10.3897/nl.38.8814

Schuurman N, Leszczynski A (2008) Ontologies for bioinformatics. Bioinformatics and biology insights. https://doi.org/10.4137/BBI.S451.

Szabolcs M, Mizsei E, Jablonski D, Vági B, Mester B, Végvári Z, Lengyel S (2017) Distribution and diversity of amphibians in Albania: new data and foundations of a comprehensive database. Amphibia-Reptilia 38: 435-448. https://doi.org/10.1163/15685381-00003126

Van Vliet K, Moore C (2016) Citizen Science Initiatives: Engaging the Public and Demystifying Science. Journal of Microbiology \& Biology Education 17: 13-16. https://doi. org/10.1128/jmbe.v17i1.1019

Westhoff V, van der Maarel E (1978) The Braun-Blanquet approach. In: Whittaker RH (Ed.) Classification of Plant Communities. Junk, The Hague, 287-399. https://doi. org/10.1007/978-94-009-9183-5_9

Wheeler QD, Knapp S, Stevenson DW, Stevenson J, Blum SD, Boom BM, Borisy GG, Buizer JL, De Carvalho MR, Cibrian A, Donoghue MJ, et al. (2012) Mapping the biosphere: exploring species to understand the origin, organization and sustainability of biodiversity. Systematics and Biodiversity 10: 1-20. https://doi.org/10.1080/14772000.2012.665095

Zeneli G, Shuka L, Bego F, Kashta L, Shumka S, Hoxha S, Diku A, Bano E, Krasniqi F (2014) National Biodiversity Strategy of Albania (2012-2020). Tirana, 146 pp. http://www.mjedisi. gov.al/al/programi/biodiversiteti-dhe-zonat-e-mbrojtura/biodiversiteti 